\title{
La Geografía en el IPGH
}

\author{
Geography in the PAIGH
}

Héctor Óscar José Pena*

Fecha de recibido: 8 de octubre de 2020

Fecha de aceptado: 29 de marzo de 2021

\section{Resumen}

La Geografía estuvo presente en la gestación del Instituto Panamericano de Geografía e Historia (IPGH), forma parte de su nombre y lo viene acompañando en su importante trayectoria institucional.

Durante noventa y dos años fue partícipe necesario en el inicial proceso de organización y desarrollo. Su evolución científica respondió a nuevos desafíos y fue adaptando su paradigma junto con las modernas tecnologías, para continuar siendo una herramienta fundamental para cumplir los objetivos de este organismo especializado de la Organización de los Estados Americanos (OEA).

Su enfoque transversal y su flexibilidad para el trabajo científico conjunto, le otorgan especial vigencia para actuar ante los grandes problemas que vive América, dentro de un mundo globalizado.

Palabras clave: Geografía, Cartografía, agenda panamericana, interdisciplinario.

\section{Abstract}

Geography was present in the gestation of the Pan American Institute of Geography and History (PAIGH), it is part of its name and has been accompanying it in its important institutional career. 
For ninety-two years it was a necessary participant in the initial organization process, developed its scientific evolution, adapted its paradigm along with modern technology, and continues to be a fundamental tool to fulfill the objectives of this specialized organization of the OAS.

Its transversal approach and its flexibility for joint scientific work give it an special validity to act in the face of the great problems that America is experiencing, within a globalized world.

Key words: Geography, Cartography, Pan-American agenda, interdisciplinary.

\section{Résumé}

\section{Géographie dans le PAIGH}

La Géographie était présente dans la gestation du IPGH, elle fait partie de son nom et l'a accompagnée dans son importante carrière institutionnelle.

Pendant quatre-vingt-douze ans, elle a été un participant nécessaire au processus d'organisation initial, a développé son évolution scientifique, a adapté son paradigme avec la technologie moderne et continue d'être un outil fondamental pour atteindre les objectifs de cette organisation spécialisée de l'OEA.

Son approche transversale et sa flexibilité pour des travaux scientifiques communs lui confèrent une validité particulière pour agir face aux grands problèmes que connaît l'Amérique, dans un monde globalisé.

Mots-clés: géographie, cartographie, agenda panaméricain, interdisciplinaire.

\section{Resumo}

\section{Geografia no IPGH}

A Geografia esteve presente na gestação do IPGH, faz parte de seu nome e a acompanha em sua importante carreira institucional.

Por noventa e dois anos, foi um participante necessário no processo inicial de organização, desenvolveu sua evolução científica, adaptou seu paradigma à tecnologia moderna e continua a ser uma ferramenta fundamental para cumprir os objetivos dessa organização especializada da OEA.

Sua abordagem transversal e sua flexibilidade para o trabalho científico conjunto conferem a ela validade especial para enfrentar os grandes problemas que a América enfrenta, em um mundo globalizado.

Palavras-chave: Geografia, Cartografia, agenda pan-american, interdisciplinar. 
Cultivar la Geografía es mostrarse ocupado en el grande arte de vivir y ser completamente feliz.

Estrabón

\section{La nueva Geografía en América}

Elegí como punto de partida para difundir esta trayectoria científica institucional, a los mediados del siglo XIX, cuando Alexander von Humboldt (1769-1879) y Carl Ritter (1798-1879) llegaron a establecer las bases científicas de la geografía moderna, definiendo los principios que la vienen rigiendo desde entonces: causalidad, localización, correlación y comparación.

Hasta ese entonces, el desarrollo de nuestra ciencia en el ámbito americano tenía un carácter descriptivo, y su contenido se sustentaba fundamentalmente en las memorias escritas por los expedicionarios que llegaron al continente por vía marítima, los demarcadores que enviaron los países europeos para dimensionar las que eran sus posesiones, los colonizadores de los distintos credos y los exploradores pioneros, todas ellas enfocadas según sus propios intereses.

Los viajes de estudio que el mismo Humboldt se autofinanció, complementado por los relevamientos de Aimé Jacques Bonpland (1773-1858) fueron ampliando el conocimiento existente sobre el paisaje de muchos países de América, aportándole además bases científicas.

Se trataba básicamente de naturalistas que recopilaron datos climáticos, registraron información hidrográfica y orográfica, reconocieron la distribución de la flora y fauna valorando el valor individual de las especies, pero también como integrantes de los ecosistemas.

Humboldt en particular bregó por la unidad del género humano y rechazaba todo tipo de discriminación. Anticipó la idea de un calentamiento de la temperatura basado en explotaciones agrícolas inadecuadas y las consecuencias perjudiciales de la deforestación que limitaba la acción moderadora de la cubierta vegetal natural. Su pensamiento quedó plasmado en más de 30 volúmenes.

Comenzó a tratar los vínculos que existen entre la naturaleza y el hombre, que constituyó una transformación substancial que se daba a la geografía hasta ese momento.

También tuvo gran influencia, por aquel entonces, las aportaciones realizadas por Jacques Elisée Reclus (1830-1905), quien defendió la unidad de la geografía con base en la explicación y la comparación. 


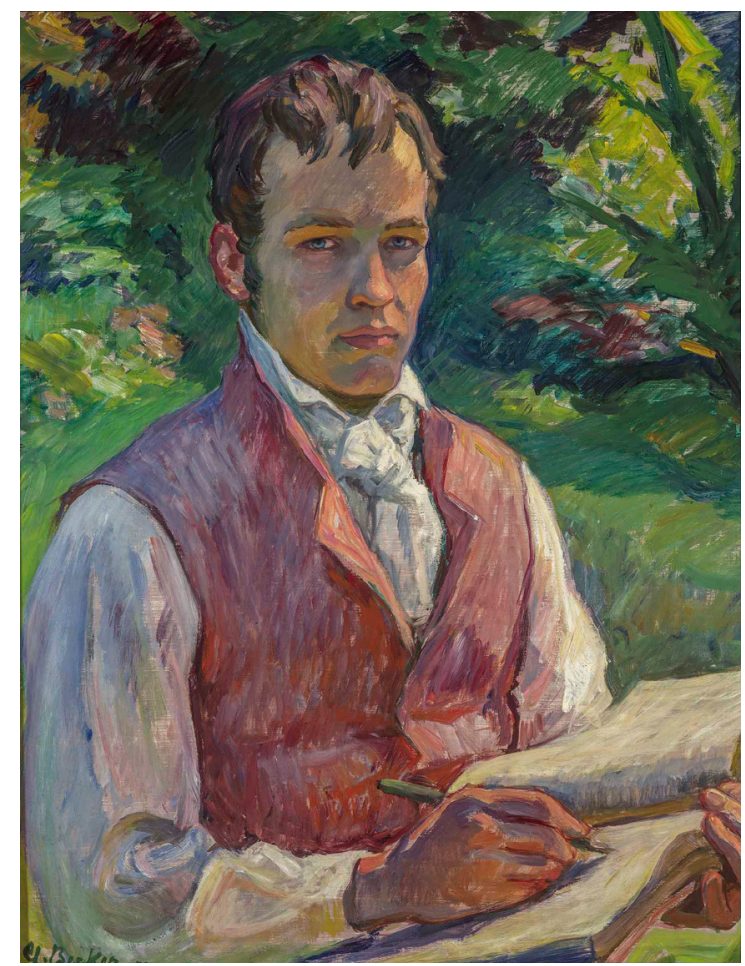

Figura 1. Alejandro von Humboldt, impulsor de la geografía en América.

Fotografía de la "Galería de Historiadores y Geógrafos de América" del IPGH.

Otra de las compilaciones de gran magnitud, localizada esta vez en la América Meridional, fue la publicada en tres tomos por Víctor Martín de Moussy (1810-1869), con el título Descripción Geográfica y Estadística de la Confederación Argentina que sufrió sucesivas actualizaciones y fue complementada con un atlas anexo. Se constituyó en una de las excluyentes fuentes de consulta durante más de medio siglo, cuando el autor hacía mucho tiempo había fallecido.

En las últimas décadas del siglo XIX aparecieron las primeras agencias cartográficas nacionales destinadas al reconocimiento y determinación de la extensión del territorio propio, para el establecimiento de puntos geodésicos de referencia, para los trabajos de levantamiento topográfico y el delineado de cartografía de inventario, necesarios para cubrir aspectos propios de la administración y la seguridad.

Generalmente estos trabajos estaban sujetos a comprobaciones técnicocientíficas por parte del Consejo Internacional de Investigaciones, integrado 
por geodestas, astrónomos y otros especialistas afines provenientes de los centros más avanzados del mundo, que tenían un costo ciertamente elevado.

Contemporáneamente comenzaron a constituirse las sociedades de Geografía que en rigor de verdad estaban conformadas por destacados cultores de distintas disciplinas que se vinculaban para el estudio de los asuntos geográficos, que iban acrecentado un interés generalizado.

En las conferencias internacionales que se venían realizando con regularidad, no obstante persistir en ellas una fuerte inclinación hacia las ciencias sistémicas, aparecían estos nuevos puntos de interés que se transformaron en una importante caja de resonancias, para las nuevas inquietudes científicas.

\section{Los inicios del IPGH}

La creación oficial del Instituto Panamericano de Geografía e Historia (IPGH) se produjo el 7 de febrero de 1928 por una resolución de la VI Conferencia Interamericana de Ministros de los Estados Americanos, celebradas en La Habana, Cuba.

Entre los objetivos acordados figuraban la coordinación y divulgación de los estudios geográficos, la cooperación de los institutos de América, la realización de investigaciones sobre la materia, la intervención en el estudio de las fronteras y la formación de un repositorio documental y de una biblioteca especializada.

La entrega por parte del gobierno mexicano del edificio que desde entonces se constituyó la sede distintiva del Instituto se realizó el 5 de mayo de 1930, coincidentemente con la conmemoración de la Batalla de Puebla.

La fecha elegida y la presencia del primer mandatario de México, evidencia la importancia que se brindó al acontecimiento.

La primera Asamblea General del novel organismo que se realizó en el año 1932, en Río de Janeiro, Brasil, dispuso pequeños cambios en el Estatuto Orgánico y prestó preferente atención a reglamentar los procedimientos y a la organización interna.

En la composición de la Primera Sección prevista para Geografía se incluían junto a la geomorfología a tres disciplinas que años después formarían parte de la Comisión de Cartografía, como eran la geodesia, la topografía y la misma cartografía, pero que en ese entonces se consideraban parte de ciencia de Estrabón.

Ese año fue muy crítico para el Instituto por una severa depresión económica mundial y obligó a reducciones presupuestarias. Se ratificó la incorporación como miembros de los países faltantes y asumió como presidente el doctor Wallace W. Atwood, fundador de la Escuela de Geografía de la Universidad de Clark, en los Estados Unidos de América. 
Con la evidente finalidad de difusión científica, recién en el año 1935 se publicó el primer título específico Evolución de la Geografía, cuyo autor fue el ingeniero Pedro Celestino Sánchez, poseedor de una gran cultura general y que actuaba como director del IPGH.

También por su iniciativa aparece, en enero del año 1941, el primer ejemplar de la Revista Geográfica, concebida originalmente como de salida trimestral.

Hasta ese momento no se habían registrado grandes cambios en la organización salvo aquellas decisiones encaminadas a explicitar las funciones previstas para los Estados Miembros y comenzar con los programas de cooperación.

Las reuniones convocadas despertaban gran interés en los círculos estatales y privados, donde estaban representadas universidades y sociedades científicas y se analizaban y sometían a consideración a un importante número de trabajos técnico-científicos.

En el mes de abril de 1941, durante la Tercera Asamblea General realizada en Lima, Perú, se crea la Comisión de Cartografía que marcó el comienzo de una nueva fase en la marcha del Instituto.

La nueva comisión estaba destinada a impulsar los levantamientos topográficos y cubrir las necesidades de contar con representaciones cartográficas confiables para el desarrollo de los países del hemisferio occidental e indispensable para abordar estudios geográficos posteriores, como lo pregonaba el influyente geógrafo Preston James.

Además de las causales enunciadas, resultó fundamental para la constitución de este nuevo componente orgánico la proximidad de una posible guerra, donde poseer un mapa de situación resultaba el documento imprescindible dentro de un potencial teatro de operaciones. El impulso decisivo fue el apoyo financiero brindado por Estados Unidos para solventar los gastos iniciales y los que se originaron en los primeros tiempos de su funcionamiento.

\section{La creación de la Comisión de Geografía}

Durante la Cuarta Asamblea General, verificada en Caracas, Venezuela, desde el 22 de agosto hasta el $1^{\circ}$ de septiembre de 1946 se creó la Comisión de Geografía.

Tuvo mucho que ver la propuesta y el fuerte respaldo que realizara en tal sentido el gobierno del Brasil, junto al importante ofrecimiento financiero para solventar los gastos del periodo de prueba de la nueva estructura.

El Secretario General del Consejo Nacional de Geografía del estado proponente fue ungido presidente interino, hasta que se completara la organización de la nueva comisión. 
La precitada reunión de Caracas contó con la asistencia más numerosa que se hubiera registrado hasta entonces. Participaron 185 representantes de 19 estados, con la particularidad de que en su casi totalidad eran especialistas en las ciencias componentes del Instituto. Desde su creación se han sucedido 12 presidencias (Figura 2).

- Dr. Christovam Leite de Castro* (1949-1952), Brasil

- Cnl. Edmundo Gastao de Cunha* (1952-1955), Brasil

- Dr. Fabio Macedo Soares Guimaraes* (1955-1965), Brasil

- Prof. Nilo Bernardes* (1955-1973), Brasil

- Dr. Harold Wood* (1973-1982), Canadá

- Dr. Clarence W. Minkel* (1982-1990), Estados Unidos

- Prof. Speridão Faisol* (1990-1997), Brasil

- Dr. Carlos Peñaherrera del Águila* (1997-2005), Perú

- Ing. Mario A. Reyes Ibarra (2005-2009), México

- Prof. Héctor Oscar José Pena (2009-2013), Argentina

- Msc. Jean W. Parcher (2013-2017), Estados Unidos

- Dra. Patricia Solís (2017-2021), Estados Unidos

Fallecidos*

Figura 2. Comisión de Geografía. Desde su creación la han sucedido 12 presidencias.

La Comisión de Geografía inicia su derrotero con una acentuada impronta académica y con el fuerte compromiso político y económico de Brasil que la lideró sin interrupciones, durante 27 años.

En la primera etapa de organización y estructuración científica fueron fundamentales entre otros, los lineamientos y orientaciones que establecieron el brasileño Christovam Leite de Castro, el argentino Federico Alberto Daus y el estadounidense Preston James.

En lo temático se destacó el impulso para realizar estudios sobre el material básico y la fuerza de trabajo disponible para el aumento de la producción, para 
mejorar la distribución de los productos agrícolas y para el establecimiento de mejores condiciones de vida.

Se estimuló la cooperación para compilar el material destinado a un atlas americano de población y colonización.

Como iniciativa práctica tuvo importancia el desarrollo de un taller de entrenamiento en las técnicas de prospección de recursos naturales.

Después de ese gran impulso inicial el desenvolvimiento de la comisión se caracterizó por el aporte mayoritario de educadores e investigadores que, salvo los subsidios para algún proyecto, no contaron con otro apoyo para sumar a su esfuerzo personal. Siempre fue una característica de la Comisión de Geografía los mínimos respaldos de entidades de gestión que son más frecuentes en otras especialidades.

\section{Los encuentros entre geógrafos}

Hemos generalizado bajo esta denominación a distintas convocatorias, previstas dentro del Estatuto Orgánico del IPGH, donde se eligen las autoridades para un nuevo periodo, se analizan los resultados del programa científico, se consideran los temas organizativos y de gestión y se establecen las principales directrices a cumplir en el mediano plazo.

Uno de los aspectos de mayor trascendencia es que se trata de las ocasiones donde participan la mayor cantidad de públicos vinculados al Instituto y se facilita el conocimiento personal y el intercambio de ideas con nuevos integrantes.

En la etapa, que hemos denominado de la consolidación institucional, a mediados del siglo pasado, resultaba habitual que se registraran participaciones que oscilaban en el medio millar de inscriptos, incluyendo altas autoridades gubernamentales, representantes diplomáticos de la región y de las fuerzas vivas locales.

Precisamente, con la nueva estructura en funcionamiento, se llevó a cabo una reunión de consulta geográfica en Washington, Estados Unidos, del 25 de julio al 4 de agosto de 1952, que tuvo la particularidad de sumar al interés de su propia convocatoria el de otros congresos de la especialidad de que se celebraron contemporáneamente, entre esa ciudad y la de Nueva York.

Fue una oportunidad no desperdiciada para que se reunieran geógrafos de distintas partes del mundo y tuvieran vivencias directas de los temas que ocupaban mayor atención.

Dentro del IPGH se adoptaron resoluciones para orientar el trabajo en climatología, problemas de la población, geografía urbana, canje de publicaciones y estímulo para trabajos conjuntos de especialistas.

Las dos reuniones siguientes se llevaron a cabo en México, desde el 25 de julio y el 6 de agosto de 1955 y en Quito, Ecuador, del 7 al 16 de enero de 1959. 
Un dato distintivo de ambas fue la participación junto a los representantes de los Estados Miembros, de observadores de Canadá, de organismos internacionales y de otras organizaciones científicas.

Abarcaron temas como los recursos naturales, climatología, estudios geográficos de las Américas, la enseñanza y textos aplicados a ella, clasificación y uso del suelo y se verificaron avances en geografía urbana y mapas de población.

Fue muy importante la colaboración brindada para la preparación uniforme de los censos para las Américas. La misma Comisión dispuso la visita de dos geógrafos a cada país y la asistencia para la mejor preparación posible de los mapas básicos, con la determinación de los límites jurisdiccionales que debían ser cubiertos por cada uno de los enumeradores del censo.

Esta tarea fue realizada en cooperación con el Instituto Interamericano de Estadística dependiente de la OEA y la ayuda permanente del gobierno de Brasil.

El corolario de estas acciones fue la ejecución del primer censo en forma conjunta de América, con evidentes mejoras de calidad y una reducción de costos para las naciones participantes, en comparación con los anteriores inventarios.

Se aprobó una declaración de principios sobre enseñanza y textos y una recomendación sobre mínimos estadísticos censales y un estudio sobre el perfeccionamiento de los mapas de censos.

Se decidió la realización de un estudio piloto de la cuenca del Pichincha, cercana a Quito, Ecuador, que combinó a especialistas de las cuatro comisiones con vistas a mejorar su desarrollo como región. Los documentos que se originaron fueron básicos para la aplicación de mejoras en el uso del suelo, en las redes de transporte y circulación y, en general, en el desarrollo regional.

En la reunión realizada en Buenos Aires, Argentina, entre el $1^{\circ}$ y el 15 de agosto de 1961, se aprobó el ingreso de Canadá como Estado Miembro, luego de haber actuado varios años como Observador. A partir de su incorporación se constituyó en un activo participante en los estudios e investigaciones geográficas y representantes de ese país lideraron por dos periodos la Comisión y se ocuparon de la edición de la revista propia de la Comisión.

La siguiente llevada a cabo en la Ciudad de Guatemala, desde el 25 de junio hasta el 10 de julio de 1965, tuvo un carácter básicamente organizativo adjudicándole carácter permanente a determinados componentes y acotando a los restantes al lapso de ejecución de las actividades encomendadas. El tema convocante consistió en el tratamiento de un proyecto sobre un atlas climatológico. 
La VIII Reunión de Consulta sobre Geografía tuvo lugar en Washington D.C. (Estados Unidos) desde el 2 al 19 de junio de 1969 y se incorporaron tres grupos de trabajo sobre Vocabulario de Geografía Urbana, Catálogo de Símbolos Geomorfológicos y Evaluación de la Enseñanza de la Geografía en América.

Se impulsó el desarrollo de centros u organizaciones geográficas y el desarrollo de estudios superiores de la ciencia, en América Latina, siguiendo modelos ya existentes.

En la IX Reunión Panamericana de Consulta sobre Geografía, llevada a cabo en la Ciudad de Panamá del 22 de abril al 9 de mayo de 1973, se adoptaron decisiones trascendentes.

La estructura de la Comisión de Geografía fue objeto de una profunda transformación para distinguir a los programas de coordinación, promoción y difusión de aquellos otros destinados a la ejecución de proyectos específicos, a cumplir en un lapso determinado.

Quedó constituida por los Comité de Geografía Urbana y Rural; sobre Problemas del Medio Ambiente; de Geomorfología; de Enseñanza y Textos y de Términos Geográficos.

Para cumplir con las tareas a término se crearon los grupos de trabajo sobre el Uso del agua en tierras áridas y semiáridas; Áreas pioneras; Efectos regionales de inversiones públicas; Interrelaciones ciudad-campo; Difusión de innovaciones; Elaboración de un texto básico sobre Metodología de investigaciones urbanas y regionales; Enseñanza de la Geografía en Centroamérica y países del Caribe; Enseñanza de la Geografía de los países andinos; Asistencia técnica sobre investigaciones geográficas y de Asesoramiento educativo.

En el año 1973, en la Asamblea de Panamá, previa propuesta de la delegación ecuatoriana, se acordó la creación oficial del Centro Panamericano de Estudios e Investigaciones Geográficas (CEPEIGE), incluyendo un aporte anual a su presupuesto y ofreciendo además el asesoramiento académico, como una de las medidas que permitiese garantizar su normal funcionamiento.

Por primera vez, en su existencia la Comisión de Geografía cambió su sede y un representante de Brasil dejó de liderarla. Durante ese periodo Angel Zarur, Nilo y Lysia Bernárdes fueron figuras destacadas.

La X Reunión se verificó en Quito, Ecuador, del 15 al 20 de agosto de 1977 y se adoptó la política científica para los próximos años. Consistió en cuatro líneas de acción, a saber: La evaluación de los recursos naturales; el estudio del problema de equilibrio entre el hombre y el medio físico; la definición de los problemas de la organización del espacio, tanto en escalas interurbanas como regionales y la contribución al mejoramiento de la enseñanza de la 
geografía en todos los niveles desde el primario al superior, incluyendo la capacitación profesional del geógrafo.

La estructura quedó reducida a los comités de Enseñanza de la Geografía; Uso del suelo y de Geografía física y al Grupo de trabajo de asistencia técnica.

El doctor Clarence W. Minkel (Estados Unidos) fue elegido presidente de la comisión en la reunión realizada en Chile en el año 1982 y reelegido por otros cuatro años en la 12a. reunión realizada en Río de Janeiro, Brasil, en 1986.

A inicios de su segundo periodo, se publicó de manera impresa el Manual de Materiales Didácticos para la Enseñanza de la Geografía a Nivel Primario, cuya autoría corresponde a las geógrafas panameñas Nidia Cardoze y Consuelo Tempone.

En la 13a. reunión realizada en Costa Rica, en el año 1990 asumió la presidencia el profesor Speridão Faisol (Brasil) y cuatro años después, por igual periodo, fue reelegido en Buenos Aires (Republica de Argentina) en el encuentro celebrado en 1993.

Se promovió la iniciativa privada en cuestiones ambientales y se analizó el impacto y desarrollo turístico. Merecieron especial consideración los espacios naturales como recursos en zonas deprimidas.

En el año 1997, en la reunión celebrada en Santiago de Chile, se encomendó la titularidad de la comisión al doctor Carlos Peñaherrera del Águila (Perú) que, posteriormente, fue reelegido por otro periodo en la 16a. reunión realizada en Santa Fe de Bogotá (Colombia), del 2001.

Se aprobó una recomendación muy importante sobre la epistemología de la Geografía y su enseñanza en América.

Otro tema de gran significación constituyó el aporte del Instituto, a través del señor John Gates, para mejor interpretar ciertos detalles de la frontera correspondiente al Tratado de Paz, celebrado entre las repúblicas de El Salvador y Honduras.

En la reunión llevada a cabo en Caracas (Venezuela) entre el 20 y el 23 de noviembre del 2005 fue electo presidente de la Comisión el ingeniero Mario Alberto Reyes Ibarra (México).

Durante el periodo se editaron con regularidad y dentro de los plazos establecidos ocho números de la Revista Geográfica.

En el año 2009, ante la renuncia del presidente y vicepresidente electos en la 17a. Reunión de Consulta, debió hacerse cargo, sin perjuicio de sus funciones, el entonces presidente del IPGH, profesor Héctor Oscar José Pena.

Fue preocupación principal recuperar el contenido epistemológico y metodológico de la ciencia. Merece destacarse el entusiasmo ante la orientación dada y el ofrecimiento de colaboración desinteresada por parte de la recordada Msc. Berta Balbín Ordaya. 
Un punto de partida necesario para el direccionamiento previsto fue conocer las características y distribución de las sociedades y centros de formación geográficos existentes en América, con la participación de todos los integrantes de la Comisión.

En el primer año de aplicación de la Agenda Panamericana 2010-2020 se orientó decididamente hacia la realización de proyectos multidisciplinarios de incumbencia regional.

Se realizó en Santo Domingo (República Dominicana), previo a la Reunión del Consejo Directivo, un simposio sobre uso racional del agua, que concluyó con importantes recomendaciones para el empleo del vital elemento.

En el mes de noviembre del 2013, en la Asamblea realizada en la ciudad de Montevideo, República Oriental del Uruguay, asumió la presidencia de la Comisión la Msc. Jean Parcher, acompañada en la vicepresidencia por la doctora Patricia Solís. En la ciudad de Panamá, en 2017, una nueva asamblea vuelve a elegir al binomio por un nuevo periodo de cuatro años, pero intercambiando roles.

Se advierte en todo momento una conducción participativa, donde se suman capacidades y que busca ampliar el sector académico sin descuidar la aplicación a proyectos interdisciplinarios de interés regional. Tal es el caso del Mapa Panamericano Integrado, para contribuir al desarrollo sostenible, a la prevención y a la mejor gestión integral.

En lo temático se priorizaron las investigaciones sobre cambio climático, riesgos naturales y antrópicos, urbanismo, análisis espacial y en lo referente a la educación geográfica, se renovó a través de una recomendación muy fundada continuar con los esfuerzos para mejorarla en todos los niveles.

Gracias a la gestión de fondos, se dictaron talleres de capacitación y se alentó la cooperación con organismos afines.

Una iniciativa de gran interés, acorde con los actuales desarrollos tecnológicos, consistió en la investigación sobre la utilización de los datos espaciales voluntarios.

\section{Distinciones y reconocimientos}

El Estatuto Orgánico vigente tiene previsto destacar institucionalmente aquellos desempeños sobresalientes. Acorde con el enfoque de este artículo, en la Figura 3 y Figura 4, se incluyen a los científicos que se desenvolvieron fundamentalmente en la Geografía. 
- Dr. Ángel Zarur* (1955), Brasil

- Prof. Ángel Rubio* (1961), Panamá

- Dr. Preston James* (1965), Estados Unidos

- Dr. Fabio de Macedo Soares Guimaraes* (1969), Brasil

- Prof. Raquel María de León* (1973), Panamá

- Prof. Nilo Bernardes* (1977), Brasil

- Dr. Paúl-Ives Denis* (1986), Canadá

- Dr. Clarence W. Minkel* (1990), Estados Unidos

- Dr. Pedro Geiger (2008), Brasil

- Dr. Mariano Zamorano Diez* (2009), República de Argentina

Fallecidos*

Figura 3. Acreedores a la Medalla Panamericana del IPGH por su trayectoria.

- Prof. Nilo Bernardes* (1986), Brasil

- Prof. Lysia Bernardes* (1988), Brasil

- Dr. Héctor F. Rucinque (1993), Colombia

- Prof. Adolfo Reinaldo Börgel Olivares (1998), Chile

- Dr. Milton Santos* (2000), Brasil

- Dr. Roberto N. Thomas (2004), Estados Unidos

- Dr. Pedro Cunill Grau (2004), Venezuela

- Dra. Edelmira González (2008), Chile

- Dr. Hugo Bodini Cruz-Carrera (2011), Chile

- Dr. Noé Pineda Portillo (2013), Honduras

- Prof. Héctor Oscar José Pena (2016), Argentina

Fallecidos*

Figura 4. Geógrafos que fueron distinguidos como Afiliados Honorarios. 


\section{Actividades de aplicación, extensión y complementación}

- Centro Panamericano de Entrenamiento para la Evaluación de los Recursos Naturales (CEPERN)

Entre los años 1954 y 1961 el Centro Panamericano de Entrenamiento para la Evaluación de Recursos Naturales realizó ocho cursos que incluían el aprendizaje de procedimientos tales como la fotogeología, el reconocimiento fotográfico de suelos y forestación aprovechable, la aeromagnetografía y la iscintilometría.

Estaban orientados al aprovechamiento de las potencialidades existentes en los países de América. Asistieron durante el periodo mencionado más de cuatrocientos científicos y técnicos de los 21 Estados Miembros del IPGH.

- Curso Internacional de Geografía Aplicada (CEPEIGE)

El Centro Panamericano de Estudios e Investigaciones Geográficas es una institución académica ecuatoriana con autonomía propia y que desde sus orígenes estuvo vinculada al Instituto.

El 3 de octubre de 1973 se suscribió un convenio de complementación entre los organismos, que dio origen al Curso Internacional de Geografía Aplicada, del que se llevan dictados 46 ediciones, ya sea presenciales o virtuales.

El primer curso se realizó bajo el título de "Nuevos Métodos para la Enseñanza e Investigación en la Geografía Moderna”. Además de otros apoyos, los doctores C. Minkel (Universidad Estatal de Michigan), T. Martinson (Universidad Ball State) y R. Thomas (Universidad Estatal de Michigan) actuaron sin remuneración. Contó con 33 participantes que representaron a 17 naciones integrantes del IPGH.

Por diversas causas no fue posible mantener las expectativas originales y debió optarse por un acuerdo sustitutivo suscrito el 11 de diciembre del 2007.

Acorde con los apoyos existentes en la actualidad y atendiendo a los avances en materia de comunicaciones, en el año 2014 se implementó un curso de educación en línea del que se llevan efectuadas cinco convocatorias.

Los cursos estuvieron a cargo de profesionales de gran prestigio y se abordaron temáticas diversas. En los últimos años se dio preferencia a los temas ambientales.

- Reunión Geográfica Internacional para Educadores de Enseñanza Primaria y Media

Entre el 6 y el 12 de febrero de 1977, se llevó a cabo en la ciudad de Panamá esta reunión que convocó a importante cantidad de docentes de todos los niveles. 
- Curso Internacional de Geografía Aplicada a Estudios Ambientales Se cumplió en la Universidad de Santiago de Chile, entre el 8 y el 31 de agosto de 1983. El IPGH tuvo oportunidad de becar a varios cursantes.

- Curso Geográfico Internacional de Análisis y Planificación Regional Se realizó en San Pedro de Macoris, República Dominicana, del 7 de enero al 3 de febrero de 1985, con la participación de cursantes becados de varios países.

- Seminario Taller sobre Métodos Geográficos para el Análisis Urbano Tuvo lugar desde el l al 6 de febrero de 1988, en Santiago de los Caballeros, República Dominicana. Resultó una avanzada metodológica sobre un tema de creciente interés, al que asistieron más de 70 cursantes.

- Primer Seminario Internacional sobre Geografía Regional

Se llevó a cabo en la Universidad Federal de Ubertlandia, en el Estado de Mina Gerais, República Federativa de Brasil, del I al 16 de septiembre de 1988.

- Cursillo de Nombres Geográficos

Impulsado por la Sección Nacional de los Estados Unidos se fueron dictando regularmente, desde el año 1995, en la gran mayoría de los Estados Miembros del IPGH, con la finalidad de normalizar la obtención, clasificación y archivo de topónimos.

Seguramente uno de sus méritos y razones de su permanencia fue la adaptación de su didáctica y contenidos curriculares a las exigencias de las nuevas tecnologías y empleo de los datos.

Otra virtud fue el de constituirse en el componente de capacitación por América del Grupo de Nombres Geográficos, que posee la Organización de las Naciones Unidas.

La versión a distancia del cursillo se mantiene actualizada para contribuir a completar eventuales necesidades de datos específicos y estandarizados que aparezcan en las capas de información de las infraestructuras geográficas.

- Congreso Internacional sobre Desastres y Ordenamiento Territorial en las Américas

Organizado por la Sección Nacional del Perú, se llevó a cabo en la Ciudad de Lima a partir del 8 de noviembre de 2010.

Participaron más de un centenar de estudiosos de la Geografía que deliberaron sobre algunos de los temas prioritarios para nuestra ciencia, alcanzando en las deliberaciones conclusiones de importancia.

- Taller de educación a distancia “Enseñar y aprender Geografía utilizando nuevas tecnologías de la comunicación y de la información” 
Por iniciativa de la Sección Nacional de la República Argentina, se dictó desde la Ciudad de Buenos Aires entre el 3 de junio hasta el 29 de setiembre de 2013. Dirigió las actividades la profesora y licenciada Graciela Cacace.

Previsto para realizarlo en 16 encuentros de no más de cuarenta educandos, recibió más de 700 solicitudes de habitantes de América.

Fue una excelente experiencia, con la posibilidad de replicarse a través de los cursantes y un buen antecedente para estimular la realización de cursos a distancia en el IPGH.

- $\quad$ 1er. Conversatorio del IPGH "Afrontando la pandemia con una mirada geoespacial"

Llevado a cabo en el mes de septiembre de 2020 con una serie de ponencias encabezada por varios especialistas en la ciencias afines de esta institución, abordaron temas enfocados a la situación del mundo en relación a la pandemia COVID-19, utilizando una plataforma de comunicación a distancia.

\section{La agenda panamericana $\mathbf{2 0 1 0 - 2 0 2 0}$}

En su etapa de consolidación científica, la Agenda Panamericana 2010-2020 resultó para el IPGH la lógica consecuencia para direccionar su quehacer en el mediano plazo, evitando posibles alternativas dilatorias y aprovechando racionalmente los recursos.

Sus contenidos responden a los aportes de los integrantes del IPGH que fueron sistematizados e integrados de acuerdo a parámetros científicos por el comité pro tempore elegido al efecto, con la coordinación del entonces Secretario General.

Los preceptos básicos perseguidos que se vienen observando consisten básicamente en propiciar el trabajo a escala continental, tender hacia temáticas de interés y promover equipos multidisciplinarios, con la participación de la mayor cantidad de nacionales de los estados miembros.

\section{La difusión}

La difusión de sus actividades, dar a conocer los avances que se van produciendo en sus ciencias específicas y el tratamiento que se brinda a los temas de interés mayoritario es fundamental para entidades como el IPGH.

En este caso los tres canales que se utilizan habitualmente son las publicaciones periódicas, las publicaciones ocasionales y las coediciones.

La tecnología digital ha permitido sumar un medio de comunicación virtual de fácil acceso, pero que a mi juicio no reemplaza a los anteriores. 


\section{Publicaciones periódicas}

La Revista Geográfica lleva publicados desde su creación en el año 1941, 161 números. Es una de las más consultadas dentro de las series del Instituto. Si bien originalmente se previó como de salida trimestral, predominó durante un tiempo prolongado la frecuencia semestral. Desde el año 2014 su edición es anual (Figura 5).

Durante sus casi 80 años de existencia se sucedieron distintas formas y criterios editoriales. Sus contenidos responden en general a proyectos de investigación que apoya el Instituto, recordatorios a figuras trascendentes de la geografía y en aportes voluntarios de integrantes del mundo universitario o de centros de investigación científica.

La Revista al igual que sus similares de las otras disciplinas para ser editada debe cumplir con exigencias de formato y calidad, cuenta con referato y está inscripta en directorios de publicaciones científicas.

La impresión y distribución de los ejemplares en soporte papel resulta cada vez más onerosa y afecta fundamentalmente el intercambio de publicaciones similares de América y el mundo. Ciertamente el intercambio virtual es una alternativa válida por el momento (https://revistasipgh.org/).

No debemos olvidar que existió un Boletín Aéreo, de salida bimestral, que debió discontinuarse con cuestiones financieras. Era un medio de lectura fácil que permitía estar actualizado en avance de proyectos, nuevas ediciones, cambios, etc. Parte de esa información puede consultarse en la página virtual institucional.

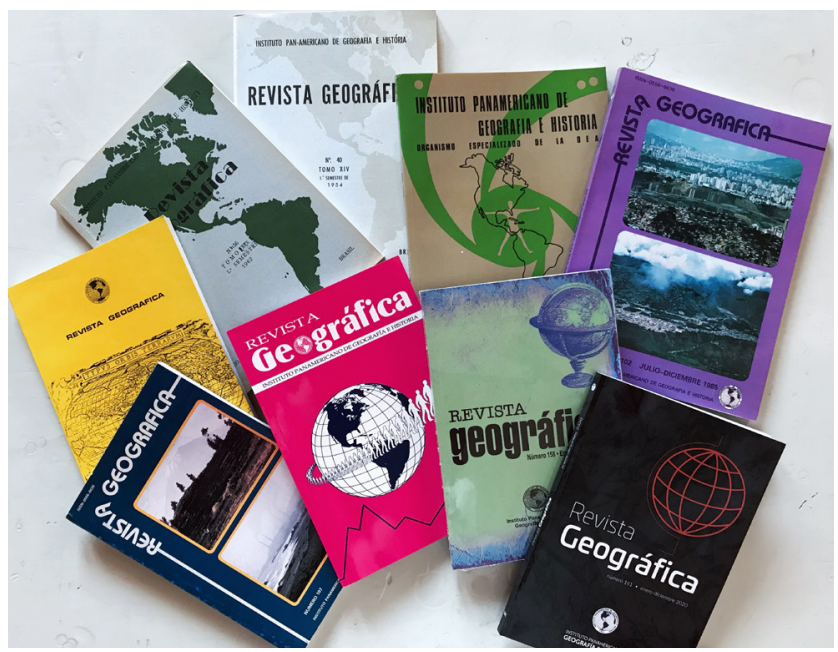

Figura 5. Revista Geográfica, una publicación tradicional. 


\section{Publicaciones ocasionales y coediciones}

Desde los inicios del Instituto, durante décadas y fundamentalmente hasta la creación de las comisiones, las publicaciones ocasionales constituyeron prácticamente el único medio utilizado para difundir las actividades del Instituto y el estado de sus ciencias constitutivas.

La línea editorial, el ritmo de edición, los autores y los distintos enfoques corresponden a los cambios que se fueron registrando en las distintas épocas.

Dentro de los más de 550 títulos editados, alrededor del $25 \%$ corresponden a temas propios o muy afines con la geografía, cursos de enseñanza, bosquejos de los países, recursos naturales mundiales, mapas físico-políticos, diccionarios, etc. (Figura 6).

Nos pareció de utilidad destacar algunas colecciones especializadas de Historia que guardan especial interés como referencias necesarias. Tal es el caso una serie de valiosas compilaciones que lideró el doctor Leopoldo Zea y están relacionadas con la actividad desarrollada por Humboldt, en territorio americano.

También son aplicables a los estudios geográficos algunos desarrollos cartográficos con contenido temático y donde se aplica simbología y toponimia específica.

Para los estudiosos de la geografía en particular creemos que merecen ser destacados algunos grupos de publicaciones que responden a proyectos con participación del IPGH:

- Ocho volúmenes del Programa de Cooperación Técnica de la OEA, realizado en los años de 1950, donde fue posible completar un importante estudio sobre los recursos naturales de las Américas. La colección conserva vigencia como fuente de consulta básica.

- Los manuales de geografía de varios de los Estados Miembros, junto con informes sobre la enseñanza de la ciencia y los materiales didácticos aplicados a ella.

- Las guías para investigadores de la geografía de numerosos países de América. Se trata de una serie de volúmenes que contienen referencias precisas sobre documentos cartográficos y bibliografía geográfica que pueden considerarse prioritarias para iniciar estudios o investigaciones sobre la ciencia.

- Los tres tomos de Geonaturalia-Geografía e Historia Natural: hacia una historia comparada. Estudio a través de Argentina, México, Costa Rica y Paraguay, permiten alcanzar una interesante visión multinacional y multidisciplinaria de cuatro países de América. 


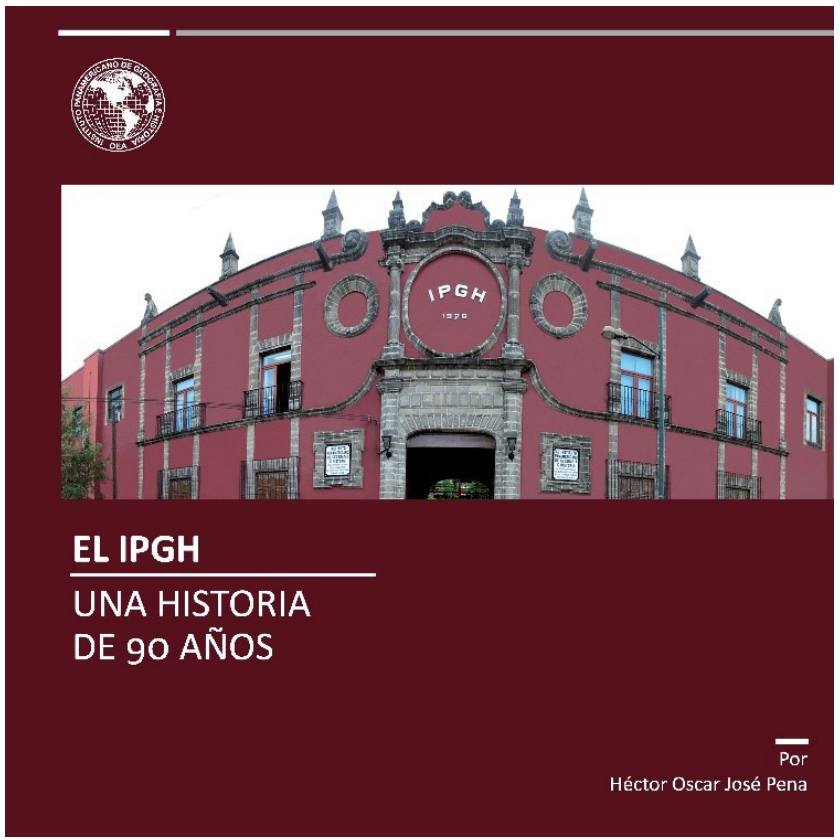

Figura 6. Testimonio de una fructífera trayectoria panamericana.

\section{Repositorios bibliográficos y cartográficos}

- Fondo Bibliográfico José Toribio Medina

Mediante un convenio con el Instituto Nacional de Antropología e Historia de México, se brindó respaldo legal al fondo bibliográfico del título que se encuentra físicamente ubicado en la Biblioteca "Guillermo Bonfil Batalla de la Escuela Nacional de Antropología e Historia, que se encarga de su resguardo y gestión.

Los ejemplares a disposición del público oscilan en los 250 000. La gran mayoría proviene de donaciones y del intercambio con diferentes instituciones, dentro y fuera del continente.

Existe un fondo reservado que incluye cerca de 400 obras, algunas únicas y en su gran mayoría originales de los siglos XVII, XVIII, XIX, XX y XXI

La digitalización de una parte de las obras permite la consulta a distancia de este importante archivo de la cultura americana

(https://mediateca.inah.gob.mx/repositorio/islandora/)

- Mapoteca Manuel Orozco y Berra.

Está instalada en un sector del gran edificio histórico donde funciona la Secretaría General del IPGH. 
Cuenta con más de 150000 documentos cartográficos que fueron impresos desde el año 1620 hasta la actualidad.

El archivo propio del Instituto, ocupa un área que incluye más de 50000 piezas correspondientes a los siglos que van del XVIII al XXI.

Gran parte de los mapas se encuentran digitalizados para minimizar el deterioro de documentos de gran valor científico, por la lógica manipulación a través del tiempo (https://mopoteca.siap.gob.mx).

\section{Algunas conclusiones}

Faltan pocos años para que el IPGH alcance el primer siglo de su existencia, al servicio de los pueblos de América, buscando a través del progreso científico un mejor entendimiento, y una buena convivencia, dentro de un espíritu de cooperación mutua.

Durante ese prolongado lapso, no frecuente en asociaciones de su tipo, se han registrado cambios significativos en su evolución institucional, en la forma de participación y hasta en los enfoques científicos.

Esas modificaciones estuvieron motivadas en transformaciones de la sociedad, en un mayor acceso a la educación en general, en adelantos científico-tecnológicos y también en las alteraciones presupuestarias para su funcionamiento.

La ocurrencia de desastres naturales o provocados casi habituales en algunos países de América, en particular el sismo que el 12 de enero de 2010 destruyó Puerto Príncipe y asoló Haití, nos reafirmó por boca de los propios afectados que la mayor ayuda que deseaban recibir del IPGH estaba en la educación, para prevenir y mitigar los daños que producían.

Nos detendremos particularmente en lo concerniente a la Geografía:

- La enseñanza de la Geografía fue y sigue siendo una de las preocupaciones prioritarias del Instituto. La misma Unión Geográfica Internacional, en una declaración difundida en el año 1992, destacó a nuestra ciencia como un poderoso instrumento educativo, tanto como un eficaz contribuyente a la educación internacional, ambiental y orientada al desarrollo. En América se registran en general avances en los enfoques, los métodos y en el empleo de modernas tecnologías, que se aplican en los diferentes niveles educativos. En casos se alcanzaron consideraciones de autonomía científica y excelencia académica, pero en otros prevalecen paradigmas con fuerte carga ideológica que afectan su desarrollo; aún existen currículos donde se la integra con otras disciplinas y se registran carencias de escuelas de formación, sobre todo en el nivel superior. El ejercicio profesional del geógrafo debe aumentar y su integración en equipos interdisciplinarios resulta cada vez más necesario. 
- El factor humano es un componente determinante en instituciones científico-técnicas, con permanente rotación de integrantes y con principales objetivos de servicio. No son pocos los casos de desempeños de excelencia por parte de estudiosos y diletantes de la Geografía, que nos dejaron testimonios de su labor y que fueron reconocidos por el Instituto. Si bien por conocimiento propio y por los antecedentes existentes del lapso analizado, no se advierte la existencia de limitaciones de género, merece destacarse la creciente y destacada participación de las geógrafas a nivel panamericano, que se viene manifestando en las últimas décadas. Bastaría considerar como ejemplo que desde el año 2013 lideran, con general aceptación, la Comisión de Geografía del IPGH.

- El cumplimiento de la Agenda Panamericana del IPGH 2010-2020, actualizada con los objetivos de desarrollo sostenible declarados por la organización de las Naciones Unidas (ONU), junto con la participación en proyectos e investigaciones de orden regional y mundial, consolida la posición del IPGH como organismo especializado de la OEA y mantiene la vigencia de sus objetivos. Tengamos presente como un ejemplo propio de la Geografía que la Organización de las Naciones Unidas para La Educación, la Ciencia y la Cultura (UNESCO) viene proponiendo desde hace años la inclusión en sus programas de enseñanza el concepto de paisaje, ínsito de nuestra ciencia, como una de las bases más sólidas para establecer una verdadera amistad entre los pueblos.

- Pensamos que el ideal de los países americanos para el siglo XXI pasa por una visión geopolítica que nace en enfoques globalizados manteniendo las identidades propias, que se acelera con los constantes desarrollos tecnológicos y se adapta a los nuevos paradigmas sociales. El IPGH pudo intervenir técnicamente en la solución pacífica de cuestiones de fronteras, que lo vincula con sus orígenes. Queda mucho por avanzar en la lucha contra el hambre y en el cuidado de la salud. Siempre resulta impostergable ocuparse seriamente del mantenimiento de la morada del hombre, donde cada día se ratifica la importancia humana y económica del conocimiento.

\section{Bibliografía}

Barriga Vargas, Rodrigo (2017). Memoria de la Secretaría General 2014-2017, publicación ocasional 553, Instituto Panamericano de Geografía e Historia, México, 97 pp.

Borrero Mutis, Santiago, (2013). Retos y logros de una década. Memoria de una gestión panamericana, publicación ocasional 548, Instituto Panamericano de Geografía e Historia, México, 75 pp. 
CEPEIGE (2008). Centro Panamericano de Estudios e Investigaciones Geográficas. Revista Geográfica, (142), 164-170, IPGH, México.

Curto, Susana I., Fritschy, Blanca A. y Pena, Héctor O. J. (2017). La Geografía en la sociedad de la información. Las academias se asoman al futuro (pp. 85-112), Buenos Aires, 296 pp.

El Instituto Panamericano de Geografía e Historia. Organismo Especializado de la OEA 1928-1978, publ. especial, México, 1978, 132 pp.

Gerlach, Arch C. (1970). Instituto Panamericano de Geografía e Historia -IPGH, publicación ocasional 314, México, 73 pp.

Pena, Héctor Óscar José (2010). El Instituto Panamericano de Geografía e Historia (IPGH). Creación, desarrollo y estrategias actuales. Boletín de GAEA Sociedad Argentina de Estudios Geográficos (pp. 77-87), Buenos Aires.

(2013). El Instituto Panamericano de Geografía e Historia. Cuatro años participando de su historia. Buenos Aires, $158 \mathrm{pp}$.

(2017). En camino. Hacia los noventa años del IPGH. Anales de la Academia Nacional de Geografía, núm. 37, 33-38, Buenos Aires.

(2018). El IPGH. Una historia de 90 años. Instituto Panamericano de Geografía e Historia, publicación ocasional 552, México, 311 pp. 